\title{
Molecular characterization in chromosome 11p15.5 related imprinting disorders Beckwith-Wiedemann and Silver-Russell syndromes
}

Young-Lim Shin $\odot$

Department of Pediatrics, Soonchunhyang University Bucheon Hospital, Soonchunhyang University College of Medicine, Bucheon, Korea

\begin{abstract}
Epigenetics deals with modifications in gene expression, without altering the underlying DNA sequence. Genomic imprinting is a complex epigenetic phenomenon that refers to parent-of-origin-specific gene expression. Beckwith-Wiedemann syndrome (BWS) and Silver-Russell syndrome (SRS) are congenital imprinting disorders with mirror opposite alterations at the genomic loci in 11p15.5 and opposite phenotypes. BWS and SRS are important imprinting disorders with the increase of knowledge of genetic and epigenetic mechanisms. Altered expression of the imprinted genes in 11p15.5, especially /GF2 and CDKN1C, affects fetal and postnatal growth. A wide range of imprinting defects at multiple loci, instead of a restricted locus, has been shown in some patients with either BWS or SRS. The development of new high-throughput assays will make it possible to allow accurate diagnosis, personalized therapy, and informative genetic counseling.
\end{abstract}

Key words: Beckwith-Wiedemann syndrome, Epigenetics, Genomic imprinting, Silver-Russell syndrome, Uniparental disomy.

\section{Introduction}

Epigenetics deals with modifications in gene expression, without altering the underlying DNA sequence. Genomic imprinting is a complex epigenetic phenomenon that refers to parent-oforigin-specific gene expression [1]. Genomic imprinting is controlled by chemical switches through genomic DNA methylation, changes in chromatin structure, post-translational histone modification, and interference with non-coding RNAs. Genomic imprinting plays an important role in embryogenesis, reproduction and growth [2]. DNA methylation is a biochemical process that transfers a methyl group $\left(\mathrm{CH}_{3}\right)$ from S-adehylmethionine to the $\mathrm{C} 5$ of a cytosine residue to form 5-methylcytosine [3]. DNA methylation of the promotor region suppresses gene expression.
Histone modification and non-coding RNA-associated gene silencing also conduce greatly to imprinting process [4]. More than 100 imprinted genes in human have been identified and most imprinted genes are arranged in clusters, or imprinting domains [5].

There are four causes of molecular alterations in imprinting disorders, which are (1) chromosomal abnormalities such as deletions, duplications or balanced rearrangements, (2) uniparental disomy (UPD), (3) epimutations and (4) point mutations (loss or gain of function) in imprinted genes. UPD is defined as the inheritance of both copies of a chromosome segment from one parent and no copy from the other parent and leads to the imbalanced expression of imprinted genes. Epimutations (aberrant methylation mark) include gain of methylation (GOM) (hyper-

Received: 24 May 2021, Revised: 17 June 2021, Accepted: 17 June 2021, Published: 30 June 2021

*Corresponding author: Young-Lim Shin, M.D. (iD https://orcid.org/0000-0002-4327-4517

Department of Pediatrics, Soonchunhyang University Bucheon Hospital, 170 Jomaru-ro, Wonmi-gu, Bucheon 14584, Korea.

Tel: +82-32-621-5407, Fax: +82-32-621-5016, E-mail: ylshin@schmc.ac.kr

Conflict of interest: I declare that I do not have any conflicts of interest.

(ㄷ) This is an open-access article distributed under the terms of the Creative Commons Attribution Non-Commercial License (http://creativecommons.org/licenses/by-nc/4.0/) which permits unrestricted non-commercial use, distribution, and reproduction in any medium, provided the original work is properly cited.

(c) Copyright 2021 by the Korean Society of Medical Genetics and Genomics 
methylation) and loss of methylation (LOM) (hypomethylation). These causes lead to imbalance gene expression including aberrant silencing of the active allele or expression of the inactive allele. One of the two parental alleles is silenced by allele-specific epigenetic modification whereas the other allele is expressed according to its parental origin [6].

Clusters contain several imprinted genes are located on chromosome 11 p15.5 region. Imprinted genes have contributed to fetal growth and development [7]. Thus, aberrant expression of these imprinted genes can be found in congenital anomalies and tumors [7]. Beckwith-Wiedemann syndrome (BWS) and Silver-Russell syndrome (SRS) are congenital imprinting disorders with mirror opposite alterations at the genomic loci in 11 p15.5 and opposite phenotypes [8]. This review focuses on the current knowledge of epigenetic and genetic alterations in BWS and SRS.

\section{The Imprinted 11p15 Region}

The 11 p15 chromosome includes two imprinted domains; the H19//GF2 domain in the telomeric region and the KCNQ1OT1/ CDKN1C domain in the centromeric region (Fig. 1). The monoallelic parent-of origin-specific expression of imprinted genes is regulated by differentially methylated regions (DMRs), also known as imprinting control regions (ICRs) [9]. Each domain is controlled by its ICR (ICR 1 in the telomeric region and ICR2 in the centromeric region) and methylated differently.

The first domain in the telomeric region contains insulin growth like factor gene 2 (IGF2), H19, and ICR1 (H19/IGF2:IGDMR) IGF2, a major fetal growth factor, expressed from the paternal allele, whereas $\mathrm{H} 19$ expressed only from the maternal allele during fetal life [7]. ICR1 is located in the intergenic region between $H 19$ and IGF2 genes and regulates imprinting through controlling the expression as a transcription insulator. ICR 1 domain is organized into two blocks of repeats (A-repeat and B-repeat elements). Several target sites for the transcriptional repressor protein called CCCTC-binding factor (CTCF) present within and around ICR1. The ICR 1 is paternally methylated, and CTCF as an insulator, cannot bind to it, thus the downstream enhancers of H19 can access /GF2 promoter and expression of IGF2. The ICR 1 is maternally unmethylated, CTCF can bind to it, and insulates IGF2 from downstream enhancers, while allowing activation of the $H 19$ promoter $[10,11]$. Therefore, IGF2 is expressed in the paternal allele and silenced in the maternal allele.

The second domain in the centromeric region contains cyclindependent kinase inhibitor $1 \mathrm{C}(C D K N 1 C)$, potassium channel
KOT family member 1 (KCNQ1), and ICR2 (KCNQ1OT1:TSS-DMR). ICR2 exists in the promoter region of KCNO1OT1 and regulates the CDKN1CIKCNO1 locus. ICR2 is methylated on the maternal allele, CDKN1C and KCNQ1 are expressed. CDKN1C gene encoding for cyclin kinase inhibitor plays a role in blocking cell proliferation. ICR2 on paternal allele is unmethylated, KCNQ10T1 is expressed. KCNQ1OT1 is a long noncoding RNA and regulates the imprinting in cis of the domain. As KCNQ1OT1 RNA expression is maternally silenced and paternally expressed, the imprinting genes of the CDKN1CIKCNQ1 locus are maternally expressed and paternally silenced [12].

In normal individuals, the paternal ICR 1 allele is methylated while the maternal ICR 1 allele is unmethylated. The paternal ICR2 allele is unmethylated while the maternal ICR2 allele is methylated. Therefore, by the paternal allele, IGF2 and KCNQ1OT1 are expressed, whereas by the maternal allele, H19 and CDKN1C are expressed. Altered expression of the imprinted genes in 11p15.5, especially /GF2 and CDKN1C, affects fetal and postnatal growth. These imprinting defects are involved in clinically opposite growth disorders, BWS and SRS [7]. Most cases of both disorders are related to opposite epigenetic or genetic abnormalities in the $11 p 15$ chromosomal region, by altering the expression of the maternally or paternally imprinted genes.

\section{Beckwith-Wiedemann Syndrome}

BWS (OMIM \# 130650) is an overgrowth syndrome, characterized by pre-and postnatal overgrowth, hemihyperplasia, macroglossia, organomegaly, neonatal hypoglycemia, hyperinsulinism, abdominal wall defects, renal abnormalities, ear creases, and increased risk of childhood tumors [13-19]. It has been known that about $10 \%$ of BWS patients could develop malignancies, e.g., hepatoblastoma and Wilms tumor in childhood [20]. BWS affects about 1 in 10,500-13,700 individuals worldwide [14,19].

BWS is associated with several epigenetic and genetic defects. A hypomethylation of maternal ICR2 can be identified in 50\%$60 \%$ of BWS cases $[15-17,21]$. This abnormality leads reduced expression of CDKN1C (a cell cycle inhibitor). Normally, ICR2 in the 11 p15 region controls the maternally expressed KCNQ1 and CDKN1C, and the paternally expressed KCNQ1OT1 gene. A hypermethylation at maternal ICR 1 is found in 5\%-10\% cases of BWS, leading to /GF2 overexpression $[15,17,22,23]$. About $20 \%$ of these methylation alterations have been associated with genetic defects within the maternal ICR1 [24,25]. Normally, /GF2 is expressed due to paternal hypermethylation of ICR 1 while $\mathrm{H} 19$ is expressed and IGF2 expression is reduced due to maternal 
Normal
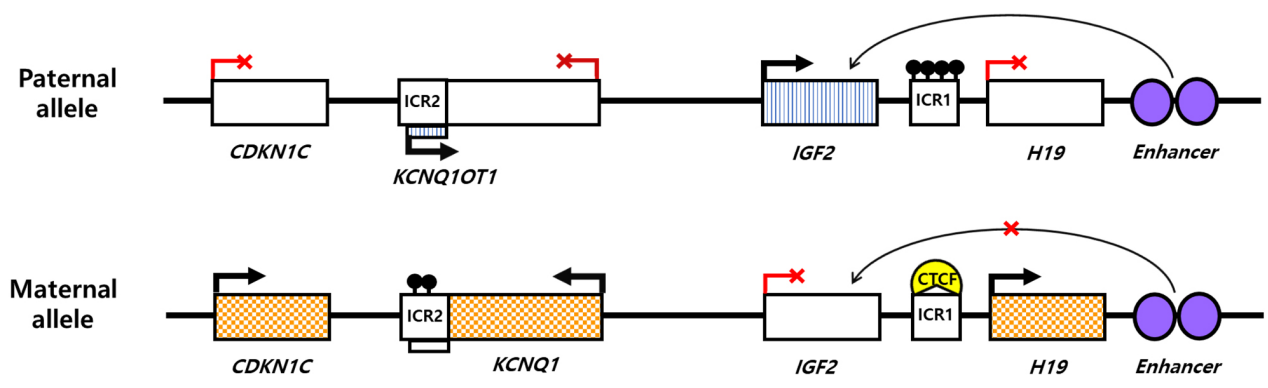

BWS

ICR1 GOM

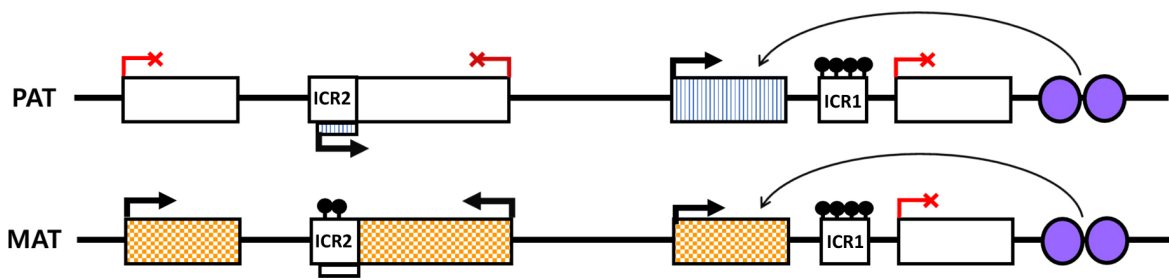

BWS

ICR2 LOM

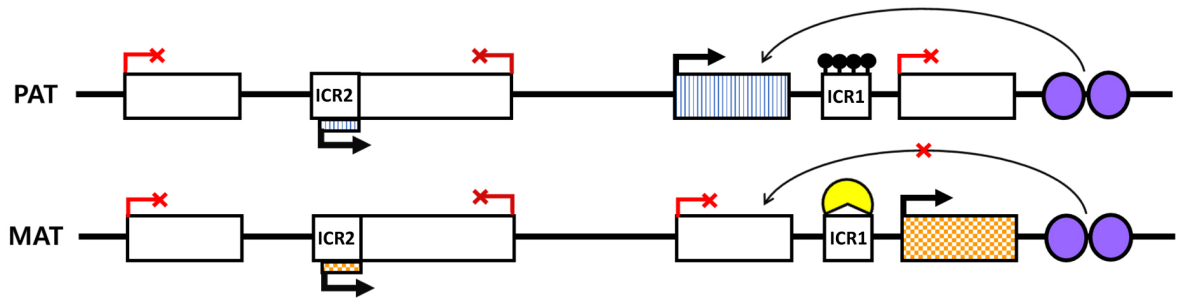

BWS

patUPD11

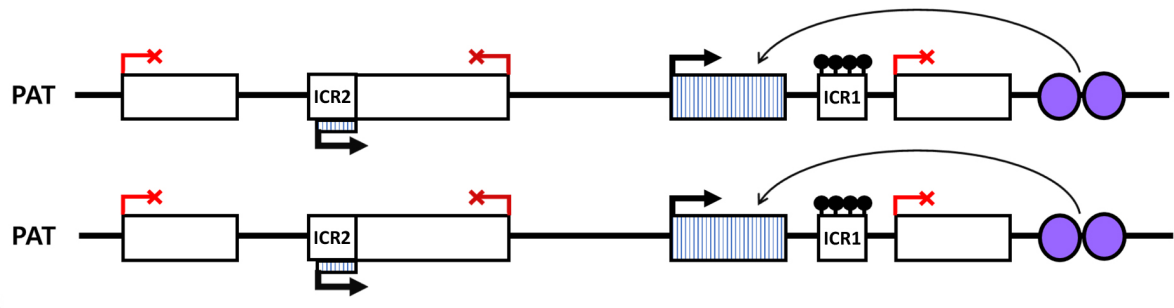

SRS

ICR1 LOM

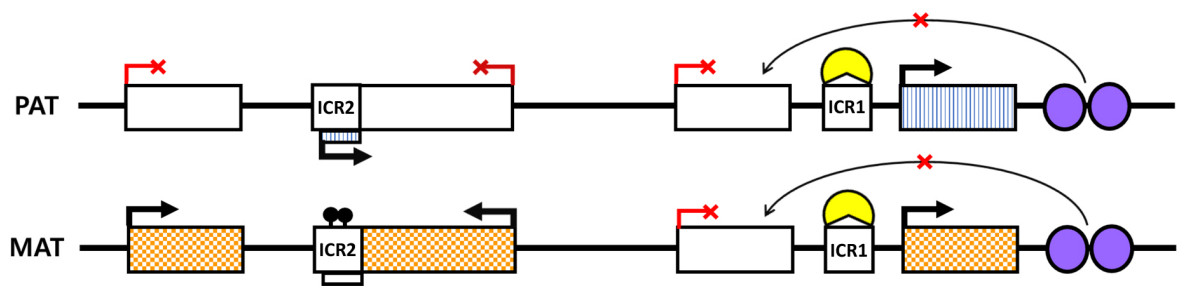

Key

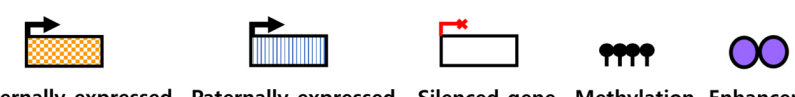

Maternally expressed Paternally expressed Silenced gene Methylation Enhancers

Fig. 1. Imprinting at the H19/IGF2 (ICR1) locus and KCNQ10T1/CDKN1C (ICR2) locus in the chromosomal region 11p15.5. Genetic and epigenetic disturbances in patients with Beckwith-Wiedemann syndrome and Silver-Russell syndrome. 
hypomethylation of ICR 1. Hypermethylation of the maternal ICR 1 accounts for biallelic expression of the /GF2 gene. Mosaic segmental paternal UPD (patUPD) in $11 \mathrm{p} 15$ region accounts for about 20\% of BWS cases [15,17,22,26]. It causes altered expression of both imprinted clusters with ICR2 LOM and ICR 1 GOM [19]. Point mutations of the maternal allele CDKN1C without methylation abnormalities are detected in 5\% cases of sporadic BWS and 50\% of familial BWS patients $[15,16,27,28]$. In about $5 \%$ of BWS cases, microdeletions involving ICR 1 have been reported and in less than $1 \%$ of BWS cases, microduplication of ICR2 are identified $[29,30]$. These molecular alterations as causes of BWS occur over-expression of paternally imprinted genes (IGF2 and KCNQ1OT1) and/or silenced expression of maternally imprinted genes (H19 and CDKN1C).

\section{Silver-Russell Syndrome}

SRS is related to heterogeneous pathogenetic mechanisms. ICR 1 hypomethylation in the $11 \mathrm{q} 15$ region is the most common epigenetic change causing SRS and accounts for $40 \%$ to $50 \%$ of SRS cases [31-33]. Hypomethylation of ICR 1 leads to the downregulation of /GF2 and the biallelic expression of H19 $[31,32]$. Maternal UPD of chromosome 7 (matUPD7), the first identified molecular cause of SRS, has been found in $5 \%$ to $10 \%$ of SRS patients $[18,31,33,34]$. In 1\% to $2 \%$ of SRS cases, a maternal duplication in $11 \mathrm{p} 15$ has been reported as a cause of SRS [35]. Other structural chromosomal aberrations including 1q21 microdeletion, 12q24 microdeletion, ring chromosome 15, and deletion 15qter have been identified in less than $2 \%$ of SRS cases [36-40]. Genomic alterations involving ICR2 resulting in GOM have been rarely described [41]. In rare cases of SRS, chromosomal structural mutations in the H19//GF2 enhancer region and paternal /GF2 loss of function mutation have been reported [42,43]. Maternal UPD11, CDKN1C gain-of-function mutation, HMGA2 mutation and PLAG 1 mutation have been published in some cases with SRS [44-48].

\section{Genotype-Phenotype Correlations in Beckwith-Wiedemann Syndrome and Silver-Russell Syndrome}

A large number of studies have been conducted to define the correlation between genotype and phenotype in BWS and SRS [19,33,49-51]. Tumor predisposition are related with BWS molecular subtypes. It has been known that the higher tumor risk is found in patients with ICR $1 \mathrm{GOM}$ and paternal UPD 11 than patients with CDKN1C mutation and ICR2 LOM $[15,19]$. The Wilms tumor prevalence in patients with ICR $1 \mathrm{GOM}$ is $21.1 \%$ and UPD subgroups is 6.2\% [52]. Hepatoblastoma development in paternal UPD cases has higher risk [52]. In BWS, exomphalos is more likely to occur in changes of the ICR2 [19]. It was indicated KCNQ1 variants predisposing to Long OT disorder associated with ICR2 LOM [50]. SRS patients with 11 p15 epimutations have more typical features than in matUPD7 [33]. Delayed neurocognitive development is more frequent in SRS patients with matUPD7 [51]. These findings contribute to allowing more accurate diagnosis, directed therapy, and individualized monitoring.

\section{Multilocus Imprinting Disturbances}

A wide range of imprinting defects at multiple loci, instead of a restricted locus, has been shown in some patients with either BWS or SRS [53]. A subset of patients with imprinting disorders exhibits aberrant methylation affecting other imprinted DMRs in addition to disease-specific locus. This condition has been named multilocus imprinting disturbance (MLID) and in particular shows frequently in BWS with ICR2 LOM, while very rare in ICR1 GOM $[19,54,55]$. Some BWS patients with MLID identify LOM or GOM at maternal and paternal ICRs, while others show a maternal hypomethylation syndrome [54-57]. In SRS, MLID has been reported less frequently [54]. Some BWS and SRS patients with MLID describe the distinct phenotypes. For instance, BWS patients with MLID show lower birth weight and less frequent macrosomia than mono-locus BWS [58]. SRS patients with MLID have growth delay and congenital abnormalities more frequently [59].

\section{Conclusion}

Various and overlapping clinical findings make it difficult to recognize and select molecular testing in both BWS and SRS. BWS and SRS are important imprinting disorders with the increase of knowledge of genetic and epigenetic mechanisms.

Molecular or cytogenetic diagnostic tests for BWS and SRS, include DNA methylation testing, sequencing analysis, copy number analysis, chromosome microarray and karyotype. Currently, methylation-specific multiplex ligation-dependent probe amplification and multiplex ligation quantitative polymerase chain reaction allow detection of DMR methylation status and copy number and are more sensitive in cases with low-level mosaicism. A single nucleotide polymorphism-based chromosome microarray analysis can detect a deletion or duplication 
and also detect segmental UPD. The development of new highthroughput assays will make it possible to better understand the pathophysiology and molecular disturbances of imprinting disorders. These improvements will contribute to accurate diagnosis, personalized therapy, and informative genetic counseling.

\section{References}

1. Demars J, Gicquel C. Epigenetic and genetic disturbance of the imprinted 11 p15 region in Beckwith-Wiedemann and Silver-Russell syndromes. Clin Genet 2012;81:350-61.

2. Elhamamsy AR. Role of DNA methylation in imprinting disorders: an updated review. J Assist Reprod Genet 2017;34:549-62.

3. Moore LD, Le T, Fan G. DNA methylation and its basic function. Neuropsychopharmacology 2013;38:23-38.

4. Maupetit-Méhouas S, Montibus B, Nury D, Tayama C, Wassef M, Kota SK, et al. Imprinting control regions (ICRs) are marked by mono-allelic bivalent chromatin when transcriptionally inactive. Nucleic Acids Res 2016;44:621-35.

5. Babak T, DeVeale B, Tsang EK, Zhou Y, Li X, Smith KS, et al. Genetic conflict reflected in tissue-specific maps of genomic imprinting in human and mouse. Nat Genet 2015;47:544-9.

6. Hanna CW, Kelsey G. The specification of imprints in mammals. Heredity (Edinb) 2014;113:176-83.

7. Azzi S, Abi Habib W, Netchine I. Beckwith-Wiedemann and RussellSilver syndromes: from new molecular insights to the comprehension of imprinting regulation. Curr Opin Endocrinol Diabetes Obes 2014;21:30-8.

8. Soellner L, Begemann M, Mackay DJ, Grønskov K, Tümer Z, Maher ER, et al. Recent advances in imprinting disorders. Clin Genet 2017;91:313.

9. Abramowitz LK, Bartolomei MS. Genomic imprinting: recognition and marking of imprinted loci. Curr Opin Genet Dev 2012;22:72-8.

10. Ulaner GA, Yang Y, Hu JF, Li T, Vu TH, Hoffman AR. CTCF binding at the insulin-like growth factor-II (IGF2)/H19 imprinting control region is insufficient to regulate IGF2/H19 expression in human tissues. Endocrinology 2003;144:4420-6.

11. Beygo J, Citro V, Sparago A, De Crescenzo A, Cerrato F, Heitmann $M$, et al. The molecular function and clinical phenotype of partial deletions of the IGF2/H19 imprinting control region depends on the spatial arrangement of the remaining CTCF-binding sites. Hum Mol Genet 2013;22:544-57.

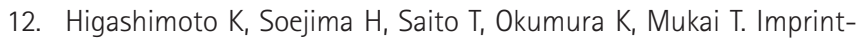
ing disruption of the CDKN1C/KCNO10T1 domain: the molecular mechanisms causing Beckwith-Wiedemann syndrome and cancer. Cytogenet Genome Res 2006;113:306-12.
13. Vora N, Bianchi DW. Genetic considerations in the prenatal diagnosis of overgrowth syndromes. Prenat Diagn 2009;29:923-9.

14. Mussa A, Russo S, De Crescenzo A, Chiesa N, Molinatto C, Selicorni A, et al. Prevalence of Beckwith-Wiedemann syndrome in North West of Italy. Am J Med Genet A 2013;161A:2481-6.

15. Cooper WN, Luharia A, Evans GA, Raza $H_{1}$ Haire AC, Grundy $R$, et al. Molecular subtypes and phenotypic expression of Beckwith-Wiedemann syndrome. Eur J Hum Genet 2005;13:1025-32.

16. Weksberg R, Shuman C, Beckwith JB. Beckwith-Wiedemann syndrome. Eur J Hum Genet 2010;18:8-14.

17. Gaston $V$, Le Bouc $Y$, Soupre V, Burglen L, Donadieu J, Oro H, et al. Analysis of the methylation status of the KCNO1OT and H19 genes in leukocyte DNA for the diagnosis and prognosis of Beckwith-Wiedemann syndrome. Eur J Hum Genet 2001;9:409-18.

18. Eggermann $T$, Perez de Nanclares $G$, Maher ER, Temple IK, Tümer $Z$, Monk $\mathrm{D}$, et al. Imprinting disorders: a group of congenital disorders with overlapping patterns of molecular changes affecting imprinted loci. Clin Epigenetics 2015;7:123.

19. Brioude F, Kalish JM, Mussa A, Foster AC, Bliek J, Ferrero GB, et al. Expert consensus document: clinical and molecular diagnosis, screening and management of Beckwith-Wiedemann syndrome: an international consensus statement. Nat Rev Endocrinol 2018;14:229-49.

20. Weksberg R, Nishikawa J, Caluseriu O, Fei YL, Shuman C, Wei C, et al. Tumor development in the Beckwith-Wiedemann syndrome is associated with a variety of constitutional molecular 11 p15 alterations including imprinting defects of KCNQ10T1. Hum Mol Genet 2001;10:2989-3000.

21. Begemann $M$, Spengler $S$, Gogiel $M$, Grasshoff $U$, Bonin $M$, Betz RC, et al. Clinical significance of copy number variations in the 11 p15.5 imprinting control regions: new cases and review of the literature. J Med Genet 2012;49:547-53.

22. Cooper WN, Curley R, Macdonald F, Maher ER. Mitotic recombination and uniparental disomy in Beckwith-Wiedemann syndrome. Genomics 2007;89:613-7.

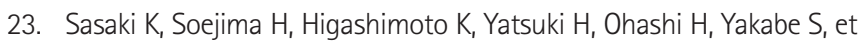
al. Japanese and North American/European patients with BeckwithWiedemann syndrome have different frequencies of some epigenetic and genetic alterations. Eur J Hum Genet 2007;15:1205-10.

24. Demars J, Shmela ME, Rossignol S, Okabe J, Netchine I, Azzi S, et al. Analysis of the IGF2/H19 imprinting control region uncovers new genetic defects, including mutations of OCT-binding sequences, in patients with 11 p15 fetal growth disorders. Hum Mol Genet 2010;19:803-14.

25. Abi Habib W, Azzi S, Brioude F, Steunou V, Thibaud N, Das Neves C, et al. Extensive investigation of the IGF2/H19 imprinting control region reveals novel OCT4/SOX2 binding site defects associated with specific 
methylation patterns in Beckwith-Wiedemann syndrome. Hum Mol Genet 2014;23:5763-73.

26. Romanelli $V$, Meneses HN, Fernández L, Martínez-Glez V, GraciaBouthelier R, F Fraga $M$, et al. Beckwith-Wiedemann syndrome and uniparental disomy $11 \mathrm{p}$ : fine mapping of the recombination breakpoints and evaluation of several techniques. Eur J Hum Genet 2011;19:416-21.

27. Eggermann $T$, Binder $G$, Brioude $F$, Maher ER, Lapunzina $P$, Cubellis MV, et al. CDKN1C mutations: two sides of the same coin. Trends Mol Med 2014;20:614-22.

28. Brioude F, Netchine I, Praz F, Le Jule M, Calmel C, Lacombe D, et al. Mutations of the imprinted CDKN1C gene as a cause of the overgrowth Beckwith-Wiedemann syndrome: clinical spectrum and functional characterization. Hum Mutat 2015;36:894-902.

29. Niemitz EL, DeBaun MR, Fallon J, Murakami K, Kugoh H, Oshimura $\mathrm{M}$, et al. Microdeletion of LIT1 in familial Beckwith-Wiedemann syndrome. Am J Hum Genet 2004;75:844-9.

30. Sparago $A$, Cerrato $F$, Vernucci $M$, Ferrero $G B$, Silengo $M C$, Riccio $A$. Microdeletions in the human H19 DMR result in loss of IGF2 imprinting and Beckwith-Wiedemann syndrome. Nat Genet 2004;36:95860.

31. Netchine I, Rossignol S, Dufourg MN, Azzi S, Rousseau A, Perin L, et al. 11 p15 imprinting center region 1 loss of methylation is a common and specific cause of typical Russell-Silver syndrome: clinical scoring system and epigenetic-phenotypic correlations. J Clin Endocrinol Metab 2007;92:3148-54.

32. Gicquel $C$, Rossignol $S$, Cabrol $S$, Houang $M$, Steunou $V$, Barbu $V$, et al. Epimutation of the telomeric imprinting center region on chromosome 11p15 in Silver-Russell syndrome. Nat Genet 2005;37:1003-7.

33. Binder $G$, Seidel $A K$, Martin DD, Schweizer $R$, Schwarze CP, Wollmann $H A$, et al. The endocrine phenotype in Silver-Russell syndrome is defined by the underlying epigenetic alteration. J Clin Endocrinol Metab 2008;93:1402-7.

34. Kotzot $D$, Schmitt $S$, Bernasconi $F$, Robinson WP, Lurie IW, llyina $H_{\text {, et }}$ al. Uniparental disomy 7 in Silver-Russell syndrome and primordial growth retardation. Hum Mol Genet 1995:4:583-7.

35. Eggermann $T$, Heilsberg AK, Bens $S$, Siebert $R$, Beygo J, Buiting $K_{1}$ et al. Additional molecular findings in 11p15-associated imprinting disorders: an urgent need for multi-locus testing. J Mol Med (Berl) 2014:92:769-77.

36. Bruce $S$, Hannula-Jouppi K, Puoskari M, Fransson I, Simola KO, Lipsanen-Nyman $M_{1}$ et al. Submicroscopic genomic alterations in Silver-Russell syndrome and Silver-Russell-like patients. J Med Genet 2010;47:816-22.

37. Spengler $S$, Begemann $M$, Ortiz Brüchle $N$, Baudis $M$, Denecke $B$, Kroisel PM, et al. Molecular karyotyping as a relevant diagnostic tool in children with growth retardation with Silver-Russell features. J Pediatr 2012;161:933-42.

38. Fuke $T$, Mizuno $S$, Nagai $T$, Hasegawa $T$, Horikawa R, Miyoshi $Y$, et al. Molecular and clinical studies in 138 Japanese patients with SilverRussell syndrome. PLoS One 2013;8:e60105.

39. Fokstuen $S$, Kotzot D. Chromosomal rearrangements in patients with clinical features of Silver-Russell syndrome. Am J Med Genet A 2014;164A:1595-605.

40. Azzi S, Salem J, Thibaud N, Chantot-Bastaraud S, Lieber E, Netchine I, et al. A prospective study validating a clinical scoring system and demonstrating phenotypical-genotypical correlations in SilverRussell syndrome. J Med Genet 2015;52:446-53.

41. Cytrynbaum C, Chong K, Hannig V, Choufani S, Shuman C, Steele L, et al. Genomic imbalance in the centromeric 11 p15 imprinting center in three families: further evidence of a role for IC2 as a cause of RussellSilver syndrome. Am J Med Genet A 2016;170:2731-9.

42. Grønskov K, Poole RL, Hahnemann JM, Thomson J, Tümer Z, Brøndum-Nielsen $K_{1}$ et al. Deletions and rearrangements of the H19/IGF2 enhancer region in patients with Silver-Russell syndrome and growth retardation. J Med Genet 2011;48:308-11.

43. Begemann $M$, Zirn $B$, Santen $G$, Wirthgen $E$, Soellner $L$, Büttel $H M$, et al. Paternally inherited IGF2 mutation and growth restriction. $\mathrm{N}$ Engl J Med 2015;373:349-56.

44. Bullman H, Lever M, Robinson DO, Mackay DJ, Holder SE, Wakeling EL. Mosaic maternal uniparental disomy of chromosome 11 in a patient with Silver-Russell syndrome. J Med Genet 2008;45:396-9.

45. Brioude F, Oliver-Petit I, Blaise A, Praz F, Rossignol S, Le Jule M, et al. CDKN1C mutation affecting the PCNA-binding domain as a cause of familial Russell Silver syndrome. J Med Genet 2013;50:823-30.

46. De Crescenzo A, Citro V, Freschi A, Sparago A, Palumbo O, Cubellis $M V$, et al. A splicing mutation of the HMGA2 gene is associated with Silver-Russell syndrome phenotype. J Hum Genet 2015;60:287-93.

47. Abi Habib W, Brioude F, Edouard T, Bennett JT, Lienhardt-Roussie $A$, Tixier $F$, et al. Genetic disruption of the oncogenic HMGA2PLAG1-IGF2 pathway causes fetal growth restriction. Genet Med 2018;20:250-8.

48. Hübner CT, Meyer R, Kenawy A, Ambrozaityte L, Matuleviciene A, Kraft $F$, et al. HMGA2 variants in Silver-Russell syndrome: homozygous and heterozygous occurrence. J Clin Endocrinol Metab 2020;105:dgaa273.

49. Wesseler K, Kraft F, Eggermann T. Molecular and clinical opposite findings in 11 p15.5 associated imprinting disorders: characterization of basic mechanisms to improve clinical management. Int J Mol Sci 2019;20:4219.

50. Valente FM, Sparago A, Freschi A, Hill-Harfe K, Maas SM, Frints SGM, et al. Transcription alterations of KCNO1 associated with imprinted 
methylation defects in the Beckwith-Wiedemann locus. Genet Med 2019;21:1808-20.

51. Wakeling EL, Brioude F, Lokulo-Sodipe O, O'Connell SM, Salem J, Bliek $J$, et al. Diagnosis and management of Silver-Russell syndrome: first international consensus statement. Nat Rev Endocrinol 2017;13:10524.

52. Mussa A, Molinatto C, Baldassarre G, Riberi E, Russo S, Larizza L, et al. Cancer risk in Beckwith-Wiedemann syndrome: a systematic review and meta-analysis outlining a novel (epi)genotype specific histotype targeted screening protocol. J Pediatr 2016;176:142-9.e1.

53. Azzi S, Rossignol S, Le Bouc $Y$, Netchine I. Lessons from imprinted multilocus loss of methylation in human syndromes: a step toward understanding the mechanisms underlying these complex diseases. Epigenetics 2010;5:373-7.

54. Court F, Martin-Trujillo A, Romanelli V, Garin I, Iglesias-Platas I, Salafsky l, et al. Genome-wide allelic methylation analysis reveals diseasespecific susceptibility to multiple methylation defects in imprinting syndromes. Hum Mutat 2013;34:595-602.

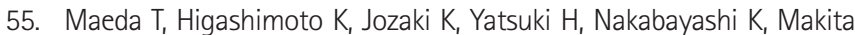
$Y$, et al. Comprehensive and quantitative multilocus methylation analysis reveals the susceptibility of specific imprinted differentially methylated regions to aberrant methylation in Beckwith-Wiedemann syndrome with epimutations. Genet Med 2014;16:903-12.

56. Boonen SE, Pörksen S, Mackay DJ, Oestergaard E, Olsen B, BrondumNielsen $K_{1}$ et al. Clinical characterisation of the multiple maternal hypomethylation syndrome in siblings. Eur J Hum Genet 2008;16:45361.

57. Sano S, Matsubara K, Nagasaki K, Kikuchi T, Nakabayashi K, Hata K, et al. Beckwith-Wiedemann syndrome and pseudohypoparathyroidism type lb in a patient with multilocus imprinting disturbance: a femaledominant phenomenon? J Hum Genet 2016;61:765-9.

58. Bliek J, Verde G, Callaway J, Maas SM, De Crescenzo A, Sparago A, et al. Hypomethylation at multiple maternally methylated imprinted regions including PLAGL1 and GNAS loci in Beckwith-Wiedemann syndrome. Eur J Hum Genet 2009;17:611-9.

59. Poole RL, Docherty LE, Al Sayegh A, Caliebe A, Turner C, Baple E, et al. Targeted methylation testing of a patient cohort broadens the epigenetic and clinical description of imprinting disorders. Am J Med Genet A 2013;161A:2174-82. 